\title{
Analyses of Multiple Factors for Determination of "Selected Patients" Who Should Receive Rechallenge Treatment in Metastatic Colorectal Cancer: a Retrospective Study from Turkey
}

\author{
Ersin Ozaslan ${ }^{1 *}$, Ayse Ocak Duran' ${ }^{1}$, Oktay Bozkurt ${ }^{1}$, Mevlude Inanc ${ }^{1}$, Mahmut \\ Ucar $^{1}$, Veli Berk ${ }^{1}$, Halit Karaca ${ }^{1}$, Ferhan Elmali², Metin Ozkan'
}

\begin{abstract}
Background: Repeating a prior chemotherapy (rechallenge therapy) is an option for selected patients with metastatic colorectal cancer, but there is very little evidence in the literature for this approach. Thus, we reviewed our registry to evaluate prognostic factors and survival of patients who received irinotecan and oxaliplatinbased regimens as rechallenge third and fourth-line therapy. Materials and Methods: Patients who received irinotecan-based or oxaliplatin-base regimen as first-line had been rechallenged with third-line or fourth-line therapy. These patients were selected from the database of Turkish mCRC registry archives between October 2006 and June 2013 and evaluated retrospectively for factors effecting progression free survival (PFS) and overall survival (OS) by the Kaplan-Meire and Cox-regression methods. Results: Thirty-nine patients were enrolled. The median duration of follow-up was 36 months (14-68 months). Thirty-one patients (76\%) died during follow-up. In terms of rechallenge treatments, 29 patients had received third-line and 10 patients had received fourth-line. Response rate (RR) was found to be $12.9 \%$, with stable disease in 19 (48.7\%) patients. The median PFS was 6 months (95\% CI=4.64-7.35 months) and the median OS was 11 months (95\% CI=8.31-13.68 months). The factors effecting survival (PFS and OS) were only being PFS after first-line chemotherapy $\geq 12$ months $(p=0.007,95 \%$ $\mathrm{CI}=\mathbf{1 . 7 5 - 3 5 . 2 2}$ and $\mathrm{p}=\mathbf{0 . 0 0 4}, \mathbf{9 5} \% \mathrm{CI}=\mathbf{1 . 4 4 - 7 . 1 1}$, , both in univariate and multivariate analyses. $\underline{\text { Conclusions: }}$ This study indicates that rechallenge treatment could be a good option as a third or later line therapy in patients who had $\geq 12$ months PFS onreceiving first line therapy.
\end{abstract}

Keywords: Metastatic colorectal - rechallenge - selected patients - survival - prognostic factors

Asian Pac J Cancer Prev, 16 (7), 2833-2838

\section{Introduction}

Colorectal cancer (CRC) is the third most common neoplasm in the world and in Turkey and the fourth most common cause of death (Haggar et al., 2009; Karaca et al., 2011). Further their incidence has increased in recent years (Jemal et al., 2010). Approximately $50 \%$ of patients with CRC present, at diagnosis, distant metastases. Since the introduction of drugs like oxaliplatin and irinotecan, the combination of these drugs with 5-Fluorouracil (5-FU) and leucovorine (LV) is considered standard chemotherapy for advanced colorectal cancers (Fuchs et al., 2007). The combination of chemotherapy with targeted biological agents such as antiepidermal growth factor receptor (anti-EGFR) and antivascular endothelial growth factor (anti-VEGF) monoclonal antibodies has increased the median OS (Hurwitz et al., 2004; Van Cutsem et al., 2009;
Peeters et al., 2010).

After the failure of palliative first-line and second-line combination chemotherapy with fluoropyrimidines (FU), oxaliplatin and irinotecan in patients with metastatic colorectal cancer (mCRC), there are much less data on the beneficial effect of systemic thrapy. Third and later line therapy options are limited for patients whose disease has progressed after they have received target therapies with the most active chemotherapy. Definition rechallenge therapy is after an intervening treatment, of the same therapy to which tumor has already proved to be resistant, as reintroduction (Tonini et al., 2013). The patients who were good responder to first-line or second-line therapy in first evaluation, are called "selected patients". Retreatment with oxaliplatin or irinotecan based regimens might be an option in selected patients. However a few studies concluded that rechallenge treatment might have some

${ }^{1}$ Department of Medical Oncology, ${ }^{2}$ Department of Biostatistics, Erciyes University Faculty of Medicine, Kayseri, Turkey *For correspondence:dr-ozaslan@hotmail.com 
activity in selected patients with mCRC (Nielsen et al., 2014).

Over the past 30 years, there has been a great interest in clinical and molecular prognostic factors in metastatic colorectal cancer (mCRC). But there is no general consensus in considering parameters as valid and reliable prognostic factors, in patients with $\mathrm{mCRC}$. The natural history of mCRC is not always the same; patients with mCRC may have various long term prognosis and respond differently to the same treatment (Divitiis et al., 2014). Here we have evaluated the role of prognostic factors in rechallenge treatment. By this way, we aim to find out the patient group for rechallange treatment.

\section{Materials and Methods}

Data were obtained from chart reviews of mCRC patients in a single oncology department in the center of Turkey, retrospectively. Patients who were treated with irinotekan or oxaliplatin based regimens in first-line therapy and who had received first-line therapy for 6 months were eligible. Thirty-nine patients diagnosed with mCRC, between October 2006 and June 2013, were treated irinotecan or oxaliplatin in combination with fluoropyrimidines \pm bevacizumab in first line therapy. And then these patients were rechallenged irinotecan or oxaliplatin in combination with fluoropyrimidines \pm bevacizumab or cetuximab in third line treatment. To monitor disease progression, tumor markers, imaging methods and clinical evaluations were used. Response evaluation was based on RECIST criteria

Table 1. The Demographic Characteristics of Patients During Rechallenge Therapy

\begin{tabular}{|c|c|c|c|}
\hline Treatment line & Total & Third-line & Fourth-line \\
\hline No. of patients & 39 & 29 & 10 \\
\hline Age (median) & 56 & 56 & 57 \\
\hline \multicolumn{4}{|l|}{ Gender } \\
\hline Male & 23 & 17 & 6 \\
\hline Female & 16 & 12 & 4 \\
\hline \multicolumn{4}{|l|}{ ECOG PS } \\
\hline 0 & 13 & 10 & 3 \\
\hline 1 & 20 & 17 & 3 \\
\hline 2 & 6 & 2 & 4 \\
\hline \multicolumn{4}{|l|}{ Tumor location } \\
\hline Colon & 26 & 20 & 6 \\
\hline Rectum & 13 & 9 & 4 \\
\hline \multicolumn{4}{|l|}{ K-RAS } \\
\hline Wild & 22 & 21 & 1 \\
\hline Mutant & 17 & 8 & 9 \\
\hline \multicolumn{4}{|l|}{ Metastasis } \\
\hline Single-site metastasis & 19 & 14 & 5 \\
\hline Multi-site metastasis & 20 & 15 & 5 \\
\hline \multicolumn{4}{|l|}{ Regimen } \\
\hline FOLFIRI & 26 & 19 & 7 \\
\hline FOLFOX/XELOX & 13 & 10 & 3 \\
\hline + Bevacizumab & 14 & 10 & 4 \\
\hline + Cetuximab & 18 & 16 & 2 \\
\hline
\end{tabular}

every 3-4 cycles chemotherapy. Toxicity was evaluated according to the National Cancer Institute (NCI) common toxicity criteria, and dose reductions or dose delaying were made according to side-effects. In case of grade 3/4 of severe adverse event, a $20 \%$ dose reduction of all cytotoxic agents was done. For statistical analyses of the study data SPSS 18.0 software was used. PFS was calculated from the date of commencing repeat irinotecan-based and oxaliplatin-based therapy to radiologic progression or death if no computed tomography results were available. PFS and OS were calculated by the Kaplan-Meier method.

An association between survival of rechallenge treatment and several factors, including age, sex, initial TNM staging (stage 2,3/stage 4), primary tumor site (colon/ rectum), adjuvant therapy status, number of distant organ metastasis (single/multipl), presence of metastasectomy, presence of K-RAS mutation, increase of CEA and CA 19-9 levels, ECOG performance status before rechallenge therapy, type of rechallenge chemotherapy (irinotecan or oxaliplatin based) and targeted therapy (bevacizumab/ cetuximab), rechallenge treatment sequence (third/fourth line) and PFS of first-line chemotherapy ( $<12$ months or $\geq 12$ months) was evaluated by the Cox-Regression method with univariate and multivariate analysis.

\section{Results}

\section{Patients characteristics}

A total of 39 patients were enrolled. The median duration of follow-up at the center was 36 months. Thirty-one patients were exitus. Baseline characteristics of patients are shown in Table 1. All patients had received third-line and fourth-line therapy which was the rechallenge treatment for mCRC. Among first-line patients, 26 patients had received irinotecan-combined regimen and 13 patients had received an oxaliplatincombined regimen. Bevacizumab was used in 31 patients in first-line chemotherapy.

In terms of rechallenge treatments, 26 patients $(66.7 \%)$ had received FOLFIRI, 8 patients had received XELOX and 5 patients had received FOLFOX regimens. Twentynine patients had received rechallenge therapy in third-line and 10 patients in fourth-line. Baseline characteristics were mostly similar among patients receiving third-line and fourth-line therapy.

\section{Toxicities}

Overall 27 patients (69\%) experienced some toxicity in third-line and forth-line therapy, usually grade $1-2$. The main toxicities were haematologic and gastrointestinal. The most common severe grade $3 / 4$ toxicities were neutropenia in eleven patients, fatigue in two patients, diarrhea in two patients, nausea-vomiting in two patients, skin eruption in one patients and anemia in one patient. Grade 3/4 toxicity is identified for the $38 \%$ of irinotecan based regimens patients and $31 \%$ of oxaliplatin based regimen patients. Dose reducement was required in these patients. No toxic death was observed. In general, both regimens were well tolerated except for neutropenia.

Univariate-multivariate analysis and survival 
Table 2. Univariate and Multivariate Prognostic Factors for Progression Free Survival (PFS) and Overall Survival (OS) of Rechallenge Therapy in mCRC

\begin{tabular}{|c|c|c|c|c|c|c|c|c|}
\hline \multirow[t]{2}{*}{ Variables } & \multicolumn{4}{|c|}{ Rechallenge PFS } & \multicolumn{4}{|c|}{ Rechallenge OS } \\
\hline & $\begin{array}{l}\text { Univariate HR } \\
\text { (95\% CI) }\end{array}$ & $\mathrm{p}$ & $\begin{array}{l}\text { Multivariate HR } \\
\qquad(95 \% \mathrm{CI})\end{array}$ & $\mathrm{p}$ & $\begin{array}{l}\text { Univariate HR } \\
(95 \% \mathrm{CI})\end{array}$ & $\mathrm{p}$ & $\begin{array}{l}\text { Multivariate HR } \\
\text { (95\% CI) }\end{array}$ & $\mathrm{p}$ \\
\hline Age (years) & $1.02(0.93-1.02)$ & 0.365 & $1.01(0.92-1.04)$ & 0.654 & $1.01(0.95-1.02)$ & 0.315 & - & - \\
\hline Gender (male/female) & $1.58(0.59-4.22)$ & 0.358 & - & - & $1.28(0.62-2.65)$ & 0.501 & - & - \\
\hline Initial stage (stage 2,3/4) & $8.82(1.16-67.02)$ & 0.035 & $7.20(0.5-102.2)$ & 0.144 & $2.68(1.12-6.36)$ & 0.025 & $2.51(0.77-8.13)$ & 0.125 \\
\hline $\begin{array}{l}\text { Primary tumor site } \\
\text { (rectum/colon) }\end{array}$ & $1.78(0.57-5.54)$ & 0.320 & $1.06(0.2-5.41)$ & 0.940 & $2.06(0.89-4.72)$ & 0.088 & - & - \\
\hline Adjuvant treatment (yes/no) & 8.82(1.16-67.02) & 0.035 & $7.20(0.5-102.2)$ & 0.144 & $2.68(1.12-6.36)$ & 0.025 & $2.51(0.77-8.13)$ & 0.125 \\
\hline Metastasectomy (yes/no) & $1.33(0.38-4.68)$ & 0.652 & $1.34(0.1-5.44)$ & 0.772 & $1.01(0.40-2.42)$ & 0.976 & $1.77(0.16-1.94)$ & 0.364 \\
\hline $\begin{array}{l}\text { First-line therapy PFS }(<12 / \\
\geq 12 \text { months })\end{array}$ & 4.99(1.41-17.67) & 0.013 & $4.99(1.4-17.67)$ & 0.013 & $2.88(1.32-6.30)$ & 0.008 & $3.17(1.28-7.84)$ & 0.012 \\
\hline ECOG PS (PS: 0-1/2) & $2.23(0.71-6.93)$ & 0.166 & $1.90(0.19-18.9)$ & 0.584 & $3.21(1.23-8.35)$ & 0.017 & $3.99(0.69-22.8)$ & 0.120 \\
\hline $\begin{array}{l}\text { Metastases (single/ } \\
\text { multipl organ) }\end{array}$ & $1.41(0.31-2.20)$ & 0.706 & $1.06(0.19-5.91)$ & 0.940 & $1.30(0.63-2.69)$ & 0.477 & $3.25(1.26-8.32)$ & 0.014 \\
\hline K-RAS (wild/mutant) & $2.42(0.87-6.66)$ & 0.087 & $3.31(0.31-35.5)$ & 0.322 & $2.14(0.99-4.65)$ & 0.052 & $3.37(1.17-9.67)$ & 0.024 \\
\hline $\begin{array}{l}\text { Rechallenge line } \\
\text { (third/fourth line) }\end{array}$ & $1.63(0.59-4.49)$ & 0.343 & - & - & $1.76(0.79-3.92)$ & 0.162 & $2.00(0.98-4.29)$ & 0.402 \\
\hline $\begin{array}{l}\text { CT regimen (irinotecan/ } \\
\text { oxaliplatin-based) }\end{array}$ & $1.79(0.66-4.84)$ & 0.248 & $1.08(0.12-6.59)$ & 0.935 & $1.80(0.82-3.95)$ & 0.138 & - & - \\
\hline $\begin{array}{l}\text { Target therapy (cetuximab/ } \\
\text { bevacizumab) }\end{array}$ & $1.14(0.51-2.54)$ & 0.310 & $1.81(0.57-5.71)$ & 0.770 & $1.14(0.51-2.54)$ & 0.731 & - & - \\
\hline CEA level (normal/high) & $3.48(0.45-26.4)$ & 0.227 & $2.68(0.80-90.4)$ & 0.582 & $2.76(0.82-9.19)$ & 0.098 & $1.54(0.98-4.29)$ & 0.654 \\
\hline CA 19-9 level (normal/high) & $2.46(0.79-7.66)$ & 0.120 & $1.40(0.11-4.41)$ & 0.714 & $2.49(1.16-5.35)$ & 0.019 & $3.70(1.17-9.67)$ & 0.008 \\
\hline
\end{tabular}

At univariate analysis results; median PFS of rechallenge treatment was longer for initial stage 2,3 CRC and received adjuvant therapy $(\mathrm{p}=0,035,95 \% \mathrm{CI}=1.16$ 67.02) and PFS of first-line chemotherapy $\geq 12$ months $(p=0.013,95 \% \mathrm{CI}=1.41-17.67)$. Similarly, median OS of rechallenge treatment was longer for initial stage 2,3 CRC and received adjuvant therapy $(\mathrm{p}=0.025,95 \% \mathrm{CI}=0.77$ 8.13 ) and longer for PFS of first-line chemotherapy $\geq$ 12 months $(\mathrm{p}=0.008,95 \% \mathrm{CI}=1.28-7.84)$. Additionally, patients who had normal range of CA 19-9 before the rechallenge treatment showed higher median OS of rechallange treatment as compared with the patients whose values were higher than the upper limit $(\mathrm{p}=0.019,95 \%$ $\mathrm{CI}=1.17-9.63)$. Also the median OS was significantly longer in patients with ECOG PS $0-1 \quad(p=0.017,95 \%$ $\mathrm{CI}=0.69-22.8)$. K-RAS mutation's contribution to median OS is shown at the univariate analysis but insignificant statistically $(\mathrm{p}=0.052,95 \% \mathrm{CI}=0.99-4.65)$.

However, at multivariate analysis, the factors of effecting survival (PFS and OS) were only being of PFS of first-line chemotherapy $\geq 12$ months $(\mathrm{p}=0.013,95 \%$ $\mathrm{Cl}=1.41-17.67$ and $\mathrm{p}=0.012,95 \% \mathrm{CI}=1.28-7.84)$. Also, the factors of positive effecting only rechallenge OS were K-RAS wild type, normal CA 19-9 level and single organ metastases $(\mathrm{p}<0.05)$ at multivariate analysis. The results are shown in Table 2.

Response rate (RR) was found $12.9 \%$, and stable disease in $19(48.7 \%)$ patients with rechallenge treatment. And clinical response rate was $61.6 \%$. Patients response and survival analyze of first-line and rechallenge treatment are shown in Table 3. Overall, median PFS was 6.0 months (95\% CI=4.64-7.35 months). Median PFS was 4.0 months (95\% CI=2.53-5.46 months) for PFS of first-line chemotherapy $<12$ months and 9.0 months (95\% CI=6.65-11.34) for PFS of first-line chemotherapy
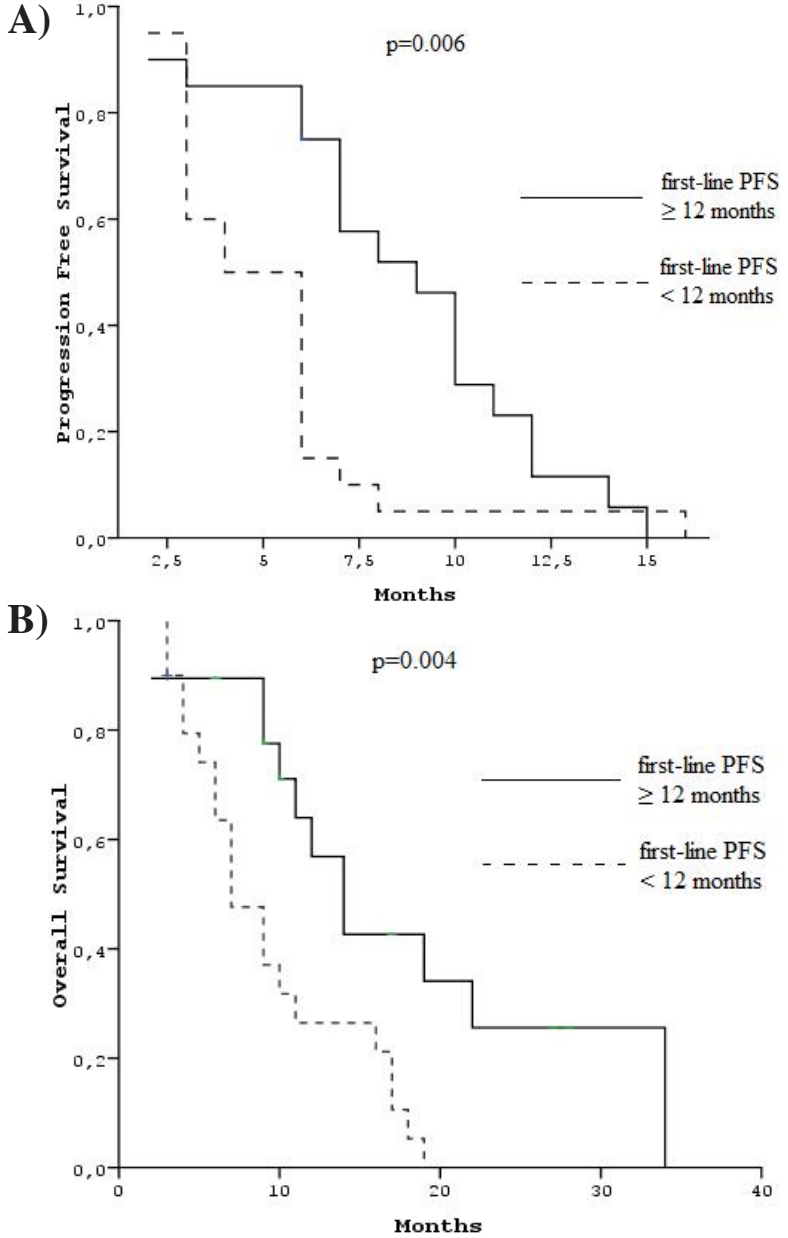

Figure 1A. The Relation between First-line Therapy PFS with Rechallenge Therapy PFS in Patients; 1 . The Relation between First-line Therapy PFS with Rechallenge Therapy OS in Patients 
Table 3. Patients Response and Survival Analyses of First-line and Rechallenge Treatment

\begin{tabular}{|c|c|c|c|c|}
\hline Patients & $\begin{array}{l}\text { First-line } \\
\text { Response }\end{array}$ & $\begin{array}{l}\text { First-line } \\
\text { PFS } \\
\text { (months) }\end{array}$ & $\begin{array}{l}\text { Rechallenge } \\
\text { therapy } \\
\text { Response }\end{array}$ & $\begin{array}{c}\text { Rechallenge } \\
\text { therapy PFS } \\
\text { (months) }\end{array}$ \\
\hline 1 & SD & 6 & PD & 3 \\
\hline 2 & SD & 6 & PR & 16 \\
\hline 3 & SD & 6 & SD & 7 \\
\hline 4 & PR & 7 & PD & 3 \\
\hline 5 & PR & 7 & PD & 4 \\
\hline 6 & PR & 7 & PD & 3 \\
\hline 7 & SD & 7 & SD & 6 \\
\hline 8 & PR & 8 & PD & 2 \\
\hline 9 & SD & 8 & SD & 6 \\
\hline 10 & SD & 8 & PD & 4 \\
\hline 11 & SD & 9 & SD & 8 \\
\hline 12 & SD & 9 & PD & 3 \\
\hline 13 & PR & 10 & PR & 12 \\
\hline 14 & PR & 10 & SD & 6 \\
\hline 15 & SD & 12 & PD & 2 \\
\hline 16 & CR & 12 & CR & 10 \\
\hline 17 & SD & 12 & SD & 7 \\
\hline 18 & PR & 12 & SD & 10 \\
\hline 19 & SD & 12 & SD & 11 \\
\hline 20 & PR & 13 & SD & 15 \\
\hline 21 & SD & 13 & SD & 14 \\
\hline 22 & CR & 14 & SD & 9 \\
\hline 23 & SD & 14 & SD & 7 \\
\hline 24 & PR & 17 & SD & 12 \\
\hline 25 & SD & 21 & PD & 6 \\
\hline 26 & PR & 21 & SD & 8 \\
\hline 27 & PR & 24 & SD & 6 \\
\hline 28 & SD & 36 & PD & 2 \\
\hline 29 & $\mathrm{CR}$ & 45 & PR & 4 \\
\hline $30 *$ & SD & 6 & SD & 6 \\
\hline $31 *$ & SD & 6 & PR & 6 \\
\hline $32 *$ & SD & 6 & PD & 3 \\
\hline $33 *$ & SD & 7 & PD & 3 \\
\hline $34 *$ & SD & 7 & SD & 6 \\
\hline $35 *$ & PR & 10 & PD & 3 \\
\hline $36^{*}$ & SD & 10 & PD & 3 \\
\hline $37 *$ & PR & 12 & SD & 10 \\
\hline $38 *$ & PR & 12 & SD & 6 \\
\hline $39 *$ & CR & 14 & SD & 7 \\
\hline
\end{tabular}

*Fourth-line rechallenge therapy (The others were third-line rechallenge therapy)

$\geq 12$ months ( $p=0.006$ ) (Figure $1 \mathrm{~A}$ ). Median OS was 11.0 months (95\% CI=8.31-13.68 months) overall, 7.0 months (95\% CI=4.45-9.54 months) for PFS of first-line chemotherapy $<12$ months and 14.0 months for PFS of first-line chemotherapy $\geq 12$ months $(95 \% \mathrm{CI}=10.45$ 17.54) $(\mathrm{p}=0.004)$ (Figure 1B).

\section{Discussion}

The relatively recent introduction of oxaliplatin, irinotecan and biologic targeted therapies (Bevacizumab, Panitumumab and Cetuximab) allowed to reach the median overall survival of 24-25 months and up today monoclonal antibodies combined with standard chemotherapy are recommended for management of mCRC (Jonker et al., 2007; Douillard et al., 2014; Schwartzberg et al., 2014). Because of the improvement in survival for mCRC patient, third and later line treatment options are needed. The role of any therapy rechallenge (chemotherapy alone, chemotherapy and biologic therapy or biologic therapy alone) is still not clear. However there are few evidences of mCRC sensitivity to any rechallenged therapy (Heinemann et al., 2014). In literature, up to date, there are few and small sized studies about rechallenge treatment.

The extreme heterogeneity in survival rate, that often emerges from the results of clinical trials probably stems from the differences in the characteristics of patients and from the prognostic factors (Kohne et al., 1998). The analysis of several studies have emphasized the importance of several clinical parameters such as age (Aslam et al., 2010), white blood cell, liver transaminases (Kemeny et al., 1983), haemoglobin (Graf et al., 1994), mean platelet volume (Tuncel et al., 2014), neutrophil/ lymphocyte ratio (Dirican et al., 2014; Wen-Zhuo et al., 2014), serum alkaline phosphatase (Chibaudel et al., 2003), serum $\gamma$-glutamyl transpeptidase (Wen-Zhuo et al., 2014), serum lactate dehydrogenase (Cetin et al., 2012), serum albumin (Tebbutt et al., 2003), tumor markers like CEA (Wen-Zhuo et al., 2014) and CA 19-9 (Katoh et al., 2008), ECOG PS (Galizia et al., 2008), primary tumor site (Bajwa et al., 2009), adjuvant chemotherapy (Katoh et al., 2008), pathological grade, number of metastatic sites (Yun et al., 2007), volume of liver metastases (Kleespies et al., 2009), metastasectomy (Settmacher et al., 2011) and state of KRAS/NRAS/BRAF mutation and PTEN loss (Tejpar et al., 2012; Yokota, 2012; Atreya et al., 2013) in the metastatic setting. Among these factors, patients with a V600E BRAF mutation have a poor prognosis which only settled in the guidelines (Bokemeyer et al., 2012).

Kohne's prognostic classification is based on ECOG PS, alkaline phosphatase level, number of metastatic sites and white blood cell count (Kohne et al., 1998). And Kobayashi et al reported prognostic scoring system which is based on regional lymph node metastasis, histologic grade, liver metastasis, lung metastasis, distant lymph node metastasis, peritoneal metastasis, and curative resection (Kobayashi et al., 2013). However, the validity and applicability of these score systems are not universally accepted for mCRC.

There is no consensus for the studies carried out until today regarding the which type of patients group should be treated with rechallange treatment in mCRC. In the studies, rechallange treatment was determined only for the patients who did not progressed at first evaluation (approximately 3 months before treatment) in first-line or second line therapy. There is no exact study which shows the interaction between the rechallange therapy of PFS \& OS and PFS of the first line therapy in the literature (Divitiis et al., 2014). Studies are mostly focused on dividing the non- metastatic colon ca into two group such as; early recurrence group (within 1 year recurrence after surgical resection) and non-early recurrence group (without 1 year recurrence after surgical resection). These studies showed that survival is longer in non-early recurrence group (Tsai et al., 2009; Huh et al., 2013; Bozkurt et al., 2014). In our mCRC study, rechallenge PFS and OS durations were significantly better in patients whose PFS is longer than 12 months in first-line therapy, 
and also this data is the first in literature about this topic. Thus, for the patients who have first-line PFS $\geq 12$ months, rechallenge therapy option should be always kept in mind for the third and later-line treatments.

ECOG PS, patients in clinical trials are also stratified according to several prognostic factors that are likely to have a significant role in influencing their survival (Divitiis et al., 2014). In our study, positive effect on OS of performance status is shown but positive effect on PFS is not shown.

In two former study (Stelzner et al., 2005; Yun et al., 2007), in which stage 4 colon cancer patients were evaluated for prognostic factors, it was reported that survival is better in patients who had received adjuvan chemotherapy. Similarly, in our study, in 12 patients, who were stage 2 or 3 at diagnose and had received adjuvant therapy, rechallenge median OS durations were significantly better $(\mathrm{p}=0.017)$.

High levels of serum CEA on diagnosis has been associated with a poor prognosis in some studies, while others have found no significant correlation between CEA and prognosis (Stelzner et al., 2005; Katoh et al., 2008; Wen-Zhuo et al., 2014). The increase of CA 19-9 has demonstrated a significantly higher frequency of metastasis and distinctly lower survival rate, making it an adverse prognostic factor for CRC patients (Katoh et al., 2008; Levy et al., 2008). Katoh et al. assessed the prognostic significance of elevated serum CA 19-9 levels. They found that patients with normal CA 19-9 lived almost twice as long as those with elevated levels (19.9 vs. 10.7 months; $p=0.001)$. Furthermore, both CEA and CA19-9 have been found to be independent and significant predictors for OS in mCRC (Mitsuyama et al., 2012). Among our study patients, in the ones, whose CA 19-9 values are high before rechallenge therapy, rechallenge OS durations were shorter and this difference was statistically significant. (normal CA 19-9: median OS 16 months, high CA 19-9: median OS 7 months, $\mathrm{p}=0.019$ ). However, there is no statistically significant difference between the CEA values and rechallenge OS.CRYSTAL and OPUS studies have evaluated the associations between tumor K-RAS mutation status and PFS, OS, and response. These studies were showed that survival of the patients with K-RAS wild type was better (Bokemeyer et al., 2012). Similarly, in our study the patients who had K-RAS wild type, PFS and OS of rechallenge therapy were longer $(\mathrm{p}=0.052$ and $\mathrm{p}=0.024)$.

In conclusion, it can be predicted that in order rechallange treatment to have a positive effect on survival; the PFS of first-line therapy should be more than 12 months, presence of ECOG PS 0-1, presence of stage 2,3 and received adjuvant therapy, presence of K-RAS mutation, presence of single organ metastases and to have normal limits of CA 19-9. Among these factors, first-line PFS duration is the most important. Because it is showed that PFS and OS of rechallenge treatment were longer in patients with first-line PFS $\geq 12$ months both at univariate and multivariate analyses. At the present time, life expectancy is increasing gradually in mCRC so in the future, the importance of rechallenge therapy will grow. Therefore, the concept of "selected patient" must be well defined. Obviously, further studies are needed to better define the prognostic information allowing them to become important criteria to select patients who will benefit from the rechallenge treatment available.

\section{References}

Aslam MI, Kelkar A, Sharpe D, et al (2010). Ten years experience of managing the primary tumours in patients with stage IV colorectal cancers. Int J Surg, 8, 305-13.

Atreya CE, Sangale Z, Xu N, et al (2013). PTEN expression is consistent in colorectal cancer primaries and metastases and associates with patient survival. Cancer Med, 2, 496-506.

Bajwa A, Blunt N, Vyas S et al (2009). Primary tumour resection and survival in the palliative management of metastatic colorectal cancer. Eur J Surg Oncol, 35, 164-7.

Bokemeyer C, Van Cutsem E, Rougier P, et al (2012). Addition of cetuximab to chemotherapy as first-line treatment for KRAS wild-type metastatic colorectal cancer: pooled analysis of the CRYSTAL and OPUS randomised clinical trials. Eur $J$ Cancer, 48, 1466-75.

Bozkurt O, Inanc M, Turkmen E, et al (2014). Clinicopathological characteristics and prognosis of patients according to recurrence time after curative resection for colorectal cancer. Asian Pac J Cancer Prev, 15, 9277-81.

Cetin B, Kaplan MA, Berk V, et al (2012). Prognostic factors for overall survival in patients with metastatic colorectal carcinoma treated with vascular endothelial growth factortargeting agents. Asian Pac J Cancer Prev, 13, 1059-63.

Chibaudel B, Tournigand C, Artru P, et al (2009). FOLFOX in patients with metastatic colorectal cancer and high alkaline phosphatase level: an exploratory cohort of the GERCOR OPTIMOX1 study. Ann Oncol, 20, 1383-6.

Dirican A, Varol U, Kucukzeybek Y, et al (2014). Treatment of metastatic colorectal cancer with or without bevacizumab: can the neutrophil/lymphocyte ratio predict the efficiency of bevacizumab? Asian Pac J Cancer Prev, 15, 4781-6.

Divitiis CD, Nasti G, Montano M, et al (2014). Prognostic and predictive response factors in colorectal cancer patients: between hope and reality. World J Gastroenterol, 20, 15049-59.

Douillard JY, Siena S, Cassidy J, et al (2014). Final results from PRIME: randomized phase III study of panitumumab with FOLFOX4 for first-line treatment of metastatic colorectal cancer. Ann Oncol, 25, 1346-55.

Fuchs CS, Marshall J, Mitchell E, et al (2007). Randomized, controlled trial of irinotecan plus infusional, bolus, or oral fluoropyrimidines in first-line treatment of metastatic colorectal cancer: results from the BICC-C study. J Clin Oncol, 25, 4779-86.

Galizia G, Lieto E, Orditura M et al (2008). First-line chemotherapy vs bowel tumor resection plus chemotherapy for patients with unresectable synchronous colorectal hepatic metastases. Arch Surg, 143, 352-35.

Graf W, Bergstrom R, Påhlman L et al (1994). Appraisal of a model for prediction of prognosis in advanced colorectal cancer. Eur J Cancer, 30, 453-7.

Haggar FA, Boushey RP(2009). Colorectal cancer epidemiology: incidence, mortality, survival, and risk factors. Clin Colon Rectal Surg, 22, 191-7.

He WZ, Jiang C, Yin CX, et al (2014). Prognostic model built on blood-based biomarkers in patients with metastatic colorectal cancer. Asian Pac J Cancer Prev, 15, 7327-31.

Heinemann V, von Weikersthal LF, Decker T, et al (2014). FOLFIRI plus cetuximab versus FOLFIRI plus bevacizumab as first-line treatment for patients with metastatic colorectal cancer (FIRE-3): a randomised, open-label, phase 3 trial. 
Lancet Oncol, 15, 1065-75.

Huh JW, Kim CH, Lim SW, et al (2013). Early recurrence in patients undergoing curative surgery for colorectal cancer: is it a predictor for poor overall survival? Int J Colorectal Dis, 28, 1143-9.

Hurwitz H, Fehrenbacher L, Novotny W, et al (2004). Bevacizumab plus irinotecan, fluorouracil, and leucovorin for metastatic colorectal cancer. N Engl J Med, 350, 2335-42.

Jemal A, Siegel R, Xu J, et al (2010). Cancer statistics. $C A$ Cancer J Clin, 60, 277-300.

Jonker DJ, O'Callaghan CJ, Karapetis CS, et al (2007). Cetuximab for the treatment of colorectal cancer. $N$ Engl $J$ Med, 357, 2040-8.

Karaca H, Berk V, Inanc M, et al (2011). Epidemiologic evaluation of the patients admitted to department of medical oncology, erciyes university, medical faculty, between 2006 and 2009. J Hlth Sci, 20, 1-8.

Katoh H, Yamashita K, Kokuba Y et al (2008). Surgical resection of stage IV colorectal cancer and prognosis. World J Surg, 32, 1130-7.

Kemeny N, Braun DW (1983). Prognostic factors in advanced colorectal carcinoma. Importance of lactic dehydrogenase level, performance status, and white blood cell count. Am $J$ Med, 74, 786-94.

Kleespies A, Fuessl KE, Seeliger H et al (2009). Determinants of morbidity and survival after elective non-curative resection of stage IV colon and rectal cancer. Int J Colorectal Dis, 24, 1097-109.

Kobayashi H, Sugihara K, Kotake K (2013). Prognostic scoring system for stage IV colorectal cancer: is the AJCC subclassification of stage IV colorectal cancer appropriate? Int J Clin Oncol, 18, 696-703.

Kohne CH, Kretzschmar A, Wils J (1998). First-line chemotherapy for colorectal carcinoma-we are making progress. Onkologie, 21, 280-9.

Levy M, Visokai V, Lipska L, et al (2008). Tumor markers in staging and prognosis of colorectal carcinoma. Neoplasma, 55, 138-42.

Mitsuyama Y, Shiba H, Haruki K, et al (2012). Carcinoembryonic antigen and carbohydrate antigen 19-9 are prognostic predictors of colorectal cancer with unresectable liver metastasis. Oncol Lett, 3, 767-8.

Nielsen DL, Palshof JA, Larsen FO, Jensen BV, Pfeifer P (2014). A systematic review of salvage therapy to patients with metastatic colorectal cancer previously treated with fluorouracil, oxaliplatin and irinotecan $+/$ - targeted therapy. Cancer Treat Rev, 40, 701-15.

Peeters M, Price TJ, Cervantes A, et al (2010). Randomized phase III study of panitumumab with fluorouracil, leucovorin, and irinotecan (FOLFIRI) compared with FOLFIRI alone as second-line treatment in patients with metastatic colorectal cancer. J Clin Oncol, 28, 4706-13.

Schwartzberg LS, Rivera F, Karthaus M, et al (2014). PEAK: a randomized, multicenter phase II study of panitumumab plus modified fluorouracil, leucovorin and oxaliplatin (mFOLFOX6) or bevacizumab plus mFOLFOX6 in patients with previously untreated,unresectable, wildtype KRAS exon 2 metastatic colorectal cancer. J Clin Oncol, 20, 2240-7.

Settmacher U, Dittmar Y, Knosel T, et al (2011). Predictors of long-term survival in patients with colorectal liver metastases: a single center study and review of the literature. Int J Colorectal Dis, 26, 967-81.

Stelzner S, Hellmich G, Koch R et al (2005). Factors predicting survival in stage IV colorectal carcinoma patients after palliative treatment: a multivariate analysis. J Surg Oncol, 89, 211-7.

Tebbutt NC, Norman AR, Cunningham D et al (2003).
Intestinal complications after chemotherapy for patients with unresected primary colorectal cancer and synchronous metastases. Gut, 52, 568-73.

Tejpar S, Celik I, Schlichting M, et al (2012). Association of KRAS G13D tumor mutations with outcome in patients with metastatic colorectal cancer treated with first-line chemotherapy with or without cetuximab. J Clin Oncol, 30, 3570-7.

Tonini G, Imperatori M, Vincenzi B, Frezza AM, Santini D (2013). Rechallenge therapy and treatment holiday: different strategies in management of metastatic colorectal cancer. $J$ Exp Clin Cancer Res, 18, 32-3.

Tsai HL, Chu KS, Huang YH, et al (2009). Predictive factors of early relapse in UICC stage I-III colorectal cancer patients after curative resection. J Surg Oncol, 100, 736-43.

Tuncel T, Ozgun A, Emirzeoglu L, et al (2014). Mean platelet volume as a prognostic marker in metastatic colorectal cancer patients treated with bevacizumabcombined chemotherapy. Asian Pac J Cancer Prev, 15, 6421-23.

Van Cutsem E, Kohne CH, Hitre E, et al (2009). Cetuximab and chemotherapy as initial treatment for metastatic colorectal cancer. $N$ Engl J Med, 360, 1408-17.

Yokota T (2012). Are KRAS/BRAF mutations potent prognostic and/or predictive biomarkers in colorectal cancers? Anticancer Agents Med Chem, 12, 163-71.

Yun HR, Lee WY, Lee WS et al (2007). The prognostic factors of stage IV colorectal cancer and assessment of proper treatment according to the patient's status. Int J Colorectal Dis, 22, 1301-10. 